\title{
El programa de atención psiquiátrica dirigido a enfermos mentales sin hogar
}

\author{
Maria Isabel Vázquez Souza \\ Servicio Madrileño de Salud \\ Hospital Clínico Universitario San Carlos
}

\begin{abstract}
Resumen
El presente artículo tiene como objetivo describir un programa de salud mental innovador que desde mayo de 2003 se viene realizando en la ciudad de Madrid. El objetivo del mismo es atender, acudiendo a su entorno, a las personas sin hogar que padecen enfermedades mentales graves y servir de puente hacia los servicios sociosanitarios de la red general a los que normalmente no tiene acceso. Se describen los modelos teóricos para esta atención (outreach, tratamiento comunitario asertivo y modelo de recuperación) y el trabajo diario que se realiza; así como, las dificultades y barreras que se enfrentan en un trabajo de estas características
\end{abstract}

Palabras claves: personas sin hogar; enfermedad mental grave; outreach; tratamiento comunitario asertivo.

\begin{abstract}
The mental health unit for the homeless mentally ill. The aim of this article is to describe an innovative mental health unit running in Madrid city since May 2003. The main purposes of this unit is to provide sociosanitary attention, going to their own enviroment- the streets-, to all those homeless persons suffering severe mental disorders. This unit provide a transitional care and engagment to mainstream social and sanitary services that usually they can't reach. It's described the theoretical models sustaining this work (outreach, Assertive Community treatment and Recovery) and also the team's daily practices. As well we described some of the challenges and barriers to face working in this particular job.
\end{abstract}

Keywords: homeless; severe mental illness; outreach; assertive comunity treatment.

$\mathrm{E}$ junio de 2003 y por mor de una confluencia de circunstancias sociales, económicas y políticas se creó (inicialmente por 2 meses) un equipo formado por dos enfermeros y una psiquiatra. La tarea de este equipo era intentar abordar a las personas que vivían en la calle y que podrían estar padeciendo enfermedades mentales graves pero que no podían acceder a tratamiento. El propósito inicial era intentar diagnosticar y dimensionar el problema, comprobar que era posible atenderles y entrevistarles en la calle y ver si accederían a tratarse. Tras los dos primeros meses se habían atendido más de 20 casos y no sólo resultaba posible hablar con ellos y entrevistarles; sino que, algunas personas sin hogar (PSH) habían iniciado tratamiento y se habían resuelto problemas médicos de potencial riesgo vital con algunos de ellos. Se creó entonces, una estructura inicial de coordinación (base de la actual) con la mayoría de los recursos municipales específicos para PSH y se propició un dialogo incipiente entre lo sanitario y lo social. Al poco tiempo, el programa había crecido con la incorporación de otros profesionales y recursos. Hoy por hoy es un programa de pleno derecho del plan de salud mental de la comunidad de Madrid.

Yo tuve la suerte, o la desgracia, de formar parte de ese primer equipo como psiquiatra y de alguna forma liderar esta iniciativa. Hoy ( 8 años después) aunque mantenemos los mismos objetivos de diagnosticar, tratar, y servir de puente con los servicios sanitarios y sociales, el programa ha cambiado. Ha variado nuestra comprensión de las personas que viven en la calle, el entendimiento de las funciones y tareas en el equipo, del funcionamiento y barreras de las redes (sociales y sanitarias) en las que nos movemos y de los cambios sociales que estamos viviendo. El tratamiento se entiende como una mejora de calidad de vida y el diagnóstico incluye lo relacional y lo social. En este artículo intento describir nuestro trabajo y dejar definidas líneas teóricas que hemos seguido. Este es un programa "artesano" y cambiante; tanto por su propio nacimiento (entre el escepticismo de la red sanitaria y el deseo de la red social), como por su crecimiento (a saltos, dependiendo de las necesidades y las circunstancias políticas y económicas) y por la plasticidad de los problemas que nos encontramos de forma conjunta con la/s sociedad/es, culturas y personas con los que convivimos. Un equipo que quiera atender a esta población ha de adaptarse al entorno donde se ubique y a las características de las personas que trabajen de forma directa e indirecta con esta población; ya sean trabajadores, pacientes o los que diseñan las políticas de 
salud. Me gustaría dejar claro que este es un trabajo que merece la pena ser hecho, a cualquier coste. Mientras continuemos viendo en la calle personas que carecen de los elementos más básicos para poder vivir, decidir y participar de forma activa en la sociedad y; mientras, la enfermedad mental sea uno de los factores de esta exclusión, estamos obligados a hacer algo. Es nuestra responsabilidad como sociales y un indicador a revisar, por los políticos y la sociedad a la hora de hablar de la calidad de la atención sociosanitaria.

Para comprender la estructura de este programa hay que describir de forma sucinta los tres elementos que se ponen en juego para llevar a cabo esta tarea: Las personas sin hogar, el sistema social y sanitario de la Comunidad de Madrid y el propio programa Personas Sin Hogar. Subjetivamente, casi todos nosotros tenemos una imagen de las personas sin hogar. Casi universales son las imágenes de un vagabundo con barba y muchas capas de ropa desastrada, con una botella, con cartones; o las de una mujer de pelo cano, sucio, con bolsas o empujando un carrito. Más allá de estas imágenes resulta complejo definir o identificar a las personas sin hogar. Existe un continuum desde una integración plena en la sociedad hasta una situación de calle, pasando por diferentes grados de vulnerabilidad y exclusión; y es imposible definir, de una forma única a las personas y a las circunstancias (externas y sobretodo internas) que facilitan este proceso. ¿Por que elegir el término personas sin hogar (PSH)?

De acuerdo con Vega Gonzalez (1996):

el término sin hogar pensamos recoge con más claridad las circunstancias en las que se desarrolla la vida de un gran número de personas... Esto es, habitualmente encuentran un "techo"- ya sea en uno de los muchos albergues existentes en nuestro país, en una casa abandonada o, incluso, en una pensión barata-, un techo ajeno y temporal, pero difícilmente encuentran un "hogar. Un "hogar" ("un lugar donde vivir y de dónde yo tengo la llave", como lo definía una persona sin hogar inglesa) desde dónde reorganizar la propia historia y proyecto de vida personal. (p.24)

Bravo Ortiz (2003) afirma que dos aspectos, al menos, tienen que concurrir. Un es la carencia de un alojamiento adecuado (en espacio y condiciones de habitabilidad) y estable (sostenible en lo económico, seguro, no peligroso). Otro es la pérdida de vínculos estables, de referencias vitales, de apoyos seguros. La marginación, la autoestigmatización y el aislamiento ${ }^{1}$.

De alguna forma, la palabra hogar, incluye un lugar no sólo lo físico, sino afectivo, un lugar de crecimiento, de creación y desarrollo de la identidad, un espacio relacional.

¿A quiénes atendemos en nuestro programa?

Personas sin hogar, preferentemente sin techo ${ }^{2}$ y crónicas, que no acuden a servicios sociales y sanitarios de la red general (ya sea porque no se les admite o por que no desean acudir a ellos), crónicas, que residen en la ciudad de Madrid, de más de 18 años y que padecen una enfermedad mental grave y crónica; con o sin consumo de tóxicos. No se aceptan todas las personas en situación de exclusión sino aquellas que carecen de alojamiento o vivienda, personas que viven en la calle o albergues o que acuden a recursos como comedores o centros de día y carecen de vivienda fija. Sobretodo los que no acceden a diferentes bienes y servicios; en concreto, a los sanitarios.
No se aceptan menores, ya que existen otros servicios para su atención. El espacio queda limitado a la ciudad de Madrid, por carecer de recursos personales y tiempo suficiente para extendernos a toda la comunidad y por ser el área de influencia del Ayuntamiento de Madrid. En caso de atender de toda la comunidad necesitaríamos pactar la intervención con todos y cada uno de los ayuntamientos y, ni siquiera existen datos claros de la necesidad de intervención. Se define patología mental grave y crónica según tres dimensiones (Rodriguez \& González Cases, 2002): Diagnóstico: Fundamentalmente psicosis: Esquizofrenias, Trastorno Bipolar, Trastornos depresivos graves, trastornos delirantes y algunos trastornos graves de la personalidad; Discapacidad: Entendiéndola en un sentido amplio como dificultad para el desenvolvimiento autónomo en diferentes áreas de la vida personal y social; Duración de la enfermedad ${ }^{3}: 2$ años.

\section{¿Porque llegan a vivir personas con enfermedad mental en la calle?}

No existe una causa necesaria y suficiente causa por la que una persona que padece una enfermedad mental grave y crónica, viva en la calle. El impacto de las causas (económicas, sociales, de enfermedad, traumáticos, físicos) suele variar según el momento y la situación vital de la persona. Su efecto no tiene por que ser sumatorio; sino que, puede tener un efecto multiplicador o casi ningún efecto. Sin embargo, la relación entre enfermedad mental grave y exclusión es muy estrecha y esto suele dar lugar a una sobrerrepresentación de PSH que padecen trastornos mentales severos. La teoría del descenso social o de la orientación apunta hacia el componente biológico como clave para explicar el porque personas que padecen esquizofrenias tienden a residir en los centros urbanos (Faris \& Dunham, 1939). Plantea que la angustia que sufren las personas se reduce más fácilmente en entornos urbanos donde es más fácil aislarse de las redes familiares y sociales. De alguna forma también explicaría la tendencia al transeúntismo y a los cambios de localización (lo que se ha dado en llamar flight síndrome) de enfermos psicóticos. Irse, cambiar de localización, desaparecer, podría ser una manera de controlar el stress asociado a un aumento de las demandas relacionales o la presión social y evitar así un episodio psicótico franco. Pero estos cambios constantes reducen la posibilidad de ser atendidos, apoyados o simplemente acompañados. Se facilitan las relaciones sociales marginales y entornos menos sanos o con pocos estímulos. Se contiene pues el malestar puntual pero con graves costes para la salud física y mental.

La biografía de las PSH suele un mayor número de eventos vitales estresantes; y enfrentan en su día a día una mayor frecuencia de situaciones ansiógenas. A esto se le añade una casi total pérdida de control de su existencia y su entorno ${ }^{4}$. En la calle, o en los albergues y recursos, los horarios (comidas, descansos...) no los definen los deseos o necesidades de las personas; lo hacen muchas veces las normas de los recursos y las decisiones de terceros. No deciden con quién conviven o con que frecuencia están expuestos a otros o a condiciones climáticas adversas. Encaran violencia y ausencia de intimidad de forma casi diaria. Este estrés incrementado (citando la hipótesis de la producción o breeder hipótesis (Martínez Hernandez, 1998) descompensa fácilmente estructuras o patologías previamente 
compensadas o situaciones traumáticas del pasado. Conductas difíciles de interpretar y, a veces, más complicadas de abordar que la propia psicosis son fruto de estas vivencias previas. Suelen existir rutinas que surgieron en otros momentos y situaciones (levantarse a determinadas horas, dormir de día) que le permitían sobrevivir o compensar síntomas de malestar o angustia pero que son fuente de conflictos o estrés en alojamientos posteriores y que resultan difíciles de modificar o de explicar.

De todos modos no podemos desligar la influencia mutua de lo social en la conducta, de lo biológico y del entorno (y del azar en todo ello). No se puede olvidar que los eventos vitales en etapas tempranas de la vida se han asociado con alteraciones en la estructura cerebral, en áreas que se han asociado con la esquizofrenia, como el hipocampo, el cuerpo calloso o la amígdala (Teicher, Andersen, \&Polcari, 2003) y las PSH suelen haber padecido un mayor número que la media y en etapas más precoces de la vida.

La asistencia sanitaria y social también contribuye a esta prevalencia. No se potencian los recursos de proximidad y políticas de prevención. Cada vez más, los huecos en el sistema (por donde se deslizan pacientes y familias) se amplían. Las personas acceden al sistema, pero no se consigue una buena continuidad de los cuidados. Los familiares y las redes de apoyo informales son quienes se encargan de evitar que los pacientes pierdan citas médicas o tomen el tratamiento. El autoestigma, el estigma y las formas rígidas de respuesta del sistema son elementos que aparecen limitando una atención continua e intensiva, sobretodo al inicio del trastorno y que aceleran la discapacidad, la desesperanza, el temor, la soledad y la desconfianza de estas personas.

\section{Sistemas social y sanitario. Asistencia a las PSH en la Comunidad de Madrid}

La asistencia a las PSH en Madrid es competencia del Ayuntamiento. Esta asistencia se basa en una red de albergues y centros de acogida, comedores, roperos, centros abiertos y equipos que trabajan en la calle. Su trabajo se apoya en modelos de búsqueda y "reinclusión" social. Su eje central son los equipos municipales del Samur Social ${ }^{5}$ y la red de albergues y centros abiertos municipales. Históricamente era una red que trabajaba desde una perspectiva de beneficencia y sostenida desde los recursos de la iglesia católica. Parte de estos recursos perviven y hay que reconocerles su labor. La red se completa con el voluntariado y Organizaciones non Governamentales (ONGs). Las competencias en atención sanitaria y política residencial para personas con discapacidad, mayores y enfermos mentales dependen de las consejerías de sanidad y la consejería de familia y servicios sociales de la comunidad de Madrid.

La asistencia a personas que padecen enfermedades mentales se realiza de forma ambulatoria en los servicios de salud mental de distrito (SSM). Estos equipos son la única puerta de entrada a los recursos residenciales y de rehabilitación del programa de atención social a personas con enfermedad mental grave y persistente. La llegada a los SSM es a través de derivación del médico de atención primaria - MAP - (médico de familia) de manera voluntaria, o desde los servicios de urgencia. Para poder acudir al MAP, se necesita disponer de una tarjeta sanitaria y para conseguir esta se necesita documentación acreditativa (DNI, NIE o Pasaporte) y estar empadronado en la comunidad de Madrid. La atención en urgencias es universal, pero no garantiza un seguimiento posterior, ni el acceso a recetas o a una aportación reducida del coste de los fármacos. Aunque la asistencia sanitaria pública está garantizada en la Constitución Española no es, en realidad, "tan" universal y gratuita. La asistencia de urgencias y los ingresos hospitalarios de problemas agudos se realiza en hospitales generales, en unidades de psiquiatría.

Los recursos de alojamiento y rehabilitación son costeados por la consejería de Familia y Asuntos sociales y la derivación se hace a través de comisiones de rehabilitación con profesionales de ambas redes. La entrada en un recurso u otro depende de los SSM a través de lo programas de continuidad de cuidado de cada centro. Esta red de apoyo social cuenta con: minirresidencias, pisos y pensiones supervisadas, centros de rehabilitación psicosocial, centros de rehabilitación laboral y equipos de apoyo socio comunitario. La gestión se realiza a través de conciertos con empresas privadas. Depende de la consejería de Familia el programa PRISEMI, de especial relevancia para la asistencia a PSH que padecen enfermedad mental. Este programa, gestionado por el grupo EXTER, se inició en 1990, en el albergue municipal con el fin de detectar e iniciar el vínculo de las personas que allí residían y padecían enfermedades mentales y acercarlas a la red de salud mental. A día de hoy tiene tres puntales: el programa FIR, que realiza las funciones de enganche y rehabilitación en el albergue; el programa de pisos, para la salida a ellos de PSH con enfermedad mental y el programa de calle, completamente integrado en el equipo y programa de atención a enfermos mentales sin hogar. Esta parte de calle conforma el subequipo de rehabilitación y costea los educadores de calle, parte de los apoyos económicos (alimentación, transporte) para personas atendidas por el programa y el alojamiento temporal en pensiones.

También dependen de esta consejería las 17 plazas de minirresidencia (6 en la de Moralzarzal y 11 en la de Santa Hortensia) a las que pueden acceder los pacientes de nuestro programa y las 30 plazas de centro de día. Destacar que tanto la de Santa Hortensia como el centro de día (ambas gestionadas por Cáritas) son atípicas por sus características. Se diferencian del resto de recursos por su baja exigencia para acceder a ella (se aceptan personas que mantienen consumo activo de tóxicos, conductas disruptivas) y en que se prestan servicios de rehabilitación y de apoyo social más amplios (ropero, lavandería, duchas, consigna, talleres remunerados, clases de castellano) que en el resto de la red. Es un recurso diseñado específicamente para facilitar la transición a la red normalizada. Existe un recurso de coordinación central que coordina la priorización de plazas o recursos de manera centralizada: oficina regional de coordinación de salud mental.

A las personas que por carecer de documentación no pueden tener una tarjeta sanitaria pero requieren de asistencia por enfermedades crónicas (físicas o psíquicas) se les puede gestionar un documento para la atención sanitaria (DAS) que garantiza su asistencia, pero no el coste de la medicación y no puede ser utilizado para acreditar su identidad. Para conseguir este documento se requiere un informe médico y un informe 
social. En el caso de nuestro programa como no es requisito el tener papeles para ser atendido, normalmente se tramita a posteriori de iniciar la atención y no es un problema conseguir el informe médico, aunque sí lo es para otras personas o equipos.

\section{El programa de atención psiquiátrica dirigido a enfermos mentales sin hogar}

Para trabajar con personas que padecen enfermedades mentales y en situación de grave exclusión hay que recordar que normalmente no acuden a los servicios habituales y que hay que ir hacia ellos y, al menos, ofertarles la atención. Esta negativa a acudir suele relacionarse con la desconfianza que tienen hacia las instituciones. Ello no se debe sólo a la psicopatología sino a experiencias previas poco útiles, francamente desagradables o traumáticas. Acaban acudiendo lugares diferentes a los habituales (ONGs, organizaciones religiosas, etc) que no están diseñadas para realizar una atención sanitaria continuada. Cuando acceden al sistema sanitario lo hacen de forma puntual (en servicios de urgencias). Sus múltiples necesidades y el problema de cómo cubrirlas de forma rápida no tiene respuesta en estos servicios creados para diagnosticar y tratar enfermedades agudas en vez de a personas y problemas crónicos. Se crea una relación incómoda. Estos servicios de alguna forma disipan su angustia o su malestar calificándoles de "problema social” y no se reconocen patologías tratables y, a veces, muy limitantes. El malestar es mutuo y la sensación es de nula interlocución

En el día a día hay que trabajar a la vez con múltiples necesidades. Las necesidades varían a cada momento y con cada persona. Normalmente, hay que priorizar unas sobre otras, por falta de tiempo, de energía o de recursos o simplemente porque se necesita atender de manera urgente algunas de ellas. Muchas veces, hay que admitir y reconocer que algunas necesidades o muchas, no se van a poder cubrir o no se pueden comprender o tolerar. Eso conlleva admitir el enfado del otro, o tu propia frustración. En cada caso y a cada momento hay que valorar donde están los déficits y, sobretodo, las fortalezas para poder progresar en el cambio de manera "natural". Es muy importante escuchar y reconocer cual es la necesidad que sitúan en primer lugar, ya que ello nos puede permitir empezar a intervenir, aunque no sea a la que los profesionales darían más importancia; por ejemplo, un paquete de tabaco antes que ir al médico. Hay que abrir espacios de diálogo. Sin embargo, el sistema crea barreras que lo defienden de esta situación tan incómoda: barreras burocráticas.

La falta de documentos impide tener atención médica o incluso teniéndola pueden carecer de MAP o este no les conoce. Se vuelven "invisibles" al sistema. Enfrentan barreras debidas a la filosofía de los servicios o a los profesionales. Desde filosofías de atención más médicas, más pro-hospitalarias se comprende mal a estas personas y los programas comunitarios, de proximidad, tienen un desarrollo irregular o escaso. La patología y la situación de exclusión son en sí mismas una barrera para acceder a tratamiento. Carecer de dinero hace difícil desplazarse al MAP o pagar la medicación; incumplen citas porque son incompatibles con los horarios o rutinas de calle (horario de comedores) y los profesionales no lo suelen saber. Entenderles desde un modelo no comunitario o de cuidado hace que todo esto sea desplazado o no incluido dentro de la patología y asistimos a buenas prácticas perfectamente inútiles ${ }^{6}$. Al trabajar con estas personas existe un cambio en la intención de la intervención: No vienen a nuestra consulta, somos nosotros los que proactivamente vamos a su encuentro y creamos la relación. Co-construimos las demandas con los pacientes y el foco se desplaza desde una petición tradicional de atención; desde una lucha contra los síntomas, hacia un dialogo de cómo alcanzar un mayor bienestar.

Desde un punto de vista teórico los modelos que permiten organizar y mantener este trabajo son el trabajo en red y los modelos de outreach, tratamiento asertivo comunitario y modelo de recuperación. Se trata de organizar los recursos de otra forma. No existen técnicas o herramientas diferentes a las que se emplean con otras personas.

\section{Trabajo en red}

El desplazamiento de la atención de los enfermos mentales graves desde los hospitales psiquiátricos a la comunidad supone enfrentar el dilema de cómo cubrir necesidades que previamente quedaban ocultas por el hospital (por ejemplo: alojamiento, ocio) El concepto de red no sólo incluye a otros servicios y programas; sino a los vínculos que se establecen entre ellos. Es vital conocer la red de servicios y todos los potenciales itinerarios entre ambos. Conocer a los equipos con los que se trabaja, su filosofía, sus horarios, sus sentimientos. En este contexto cobra todo su significado la coordinación.

La coordinación intra e interequipos es una herramienta básica y una de las más complejas a utilizar. El objetivo de una buena coordinación es conseguir continuidad asistencial y que las cosas funcionen de manera eficiente. Para coordinar en el caso de las PSH hay que buscar formas y elementos comunes del lenguaje (social y sanitario, de organizaciones religiosas y no religiosas, de ONGs), crear espacios para las coordinaciones (físicos, virtuales) y dinámicas que faciliten la recogida y el feedback de la/s intervenciones. En el caso de las PSH las redes con las que hablar son amplias, diversas e incluso inhabituales. Es importante no olvidarse que el elemento central de la coordinación es el paciente/usuario/cliente. Hay que mantenerse alerta ante la posibilidad de formas de coordinación perversa (todos actuando con la persona pero sin interactuar entre sí) o ecléctica (todos interactuando entre si y con la persona pero sin su opinión real) y a los riesgos éticos y legales de banalizar la información. Compartir más información de la precisa vulnera la intimidad de la persona, la confidencialidad de la relación y socava la confianza en la misma. No se necesita saber si una persona padece una esquizofrenia para tramitar un DNI, pero si podría ser relevante saber que en ese momento le cuesta tolerar la compañía de varias personas o los ruidos por pseudoalucinaciones. Saberlo permite ir a una comisaría menos saturada o intentar coordinar previamente con la policía o no enfadarte porque no acuda a la cita.

\section{Outreach: Modelo de búsqueda activa}

Trabajar en calle es aprender a desarrollar relaciones seguras que permitan un acercamiento y posterior abordaje terapéutico sin un encuadre, físico o temporal o de rol. El modelo de búsqueda activa se ha señalado como uno de los modelos teóricos 
clave. Se basa en las teorías ecológicas y del desarrollo (Levy, 1998). Para explicarlo se subdivide en fases que, en realidad, se entrelazan y mezclan sin que haya una progresión lineal:

Preenganche. Incluye desde recoger cualquier información que nos pueda facilitar hablar con la persona hasta observar su entorno o convertirse en algo o alguien que ven todos los días. Puede ser tan largo como se precise, el objetivo de esta fase es facilitar el inicio de un proceso de comunicación y la valoración de los problemas en un ambiente de seguridad para el paciente y el/los profesionales encargados de esta fase. Es visitarles y realizar acciones tan simples como un saludo o una sonrisa sin acercamiento o una comunicación verbal si no lo permite. Es abrir un hueco que permita vencer la desconfianza inicial.

Una vez conseguido esto la fase siguiente es de Enganche, de intentar desarrollar una alianza terapéutica. Supone conocer el lenguaje del paciente, sus habilidades y limitaciones y respetar la relación limitando el desarrollo de conductas disruptivas. En esta fase la disponibilidad y la capacidad de responder a las demandas del paciente, para poder reformularlas en objetivos terapéuticos, son lo más esencial.

Desde un perspectiva más relacional Brandt (2003) denomina estas etapas: fase de contacto, iniciar la relación desde los contactos y visitas repetidas; fase de clarificación, la base de esta fase es el conocimiento entre ambos profesional-persona, de aprendizaje mutuo del lenguaje, de las necesidades y los deseos; y, fase de Integración. Señala que se debe de iniciar la ayuda por aquello que sea más sencillo, un acompañamiento por ejemplo. Propone luego continuar trabajando mientras se van concretando necesidades y objetivos que permitan mejorar su calidad de vida. En el modelo tradicional de outreach aquí se inicia una fase de contrato e implementación del contrato. O lo que es lo mismo pactar los objetivos a conseguir con el paciente y llegar su consecución y luego, mantener y consolidarlos.

En estas fases lo importante es ajustar las expectativas con un "optimismo realista". Destacando de nuevo lo relacional, Brandt habla de una fase de apoyo (continuar el apoyo, aunque se haya solucionado lo que inicialmente se pretendía) y una fase de conclusión. Incluye retirarse a tiempo y asegurarse que no se pierda lo ganado. Puede ser una fase infinitamente larga, manteniendo contactos informales (por ejemplo, en un café una vez al año) pero suficientes. Chinman, Rosenheck y Lam (1999) afirman que este es el primer paso para establecer un programa de continuidad de cuidados y más tarde, una rehabilitación más estructurada.

\section{Programas de continuidad de cuidados.}

\section{Tratamiento asertivo comunitario (TAC). Outreach asertivo}

Los programas de continuidad de cuidados son una amplia gama de formas de intervenir a largo plazo que tienen como foco de atención los enfermos mentales crónicos (Dixon, 2000; Test \& Stein, 1976). Aparecen como una respuesta a la desistitucionalización en los EEUU y pretenden minimizar la fragmentación de servicios por medio de una figura clave (gestor de casos. En el momento actual, los programas modificados de TAC o de Outreach Asertivo son los que se aplican más comúnmente a PSH. Aunque se basan en los modelos de TAC la fidelidad es variable (Wright, Burns, James, \&Billings, 2003). En estos servicios se definen tres puntos básicos. Una ratio baja pacientes: profesionales (10-15:1), incluir profesiones diferentes dentro del staff del equipo (clásicamente trabajadora social, psiquiatra, enfermera), y ser capaces de proveer tratamiento comunitario a largo plazo, a personas afectas de enfermedad mental grave. El equipo multidisciplinar debe proveer toda la atención, existiendo una corresponsabilidad en ello de todo el equipo, no de un único profesional. Se intenta evitar las hospitalizaciones, hacer la mayoría de las actuaciones terapéuticas y entrenamientos en un entorno "real", a tiempo real, incluidas las intervenciónes en crisis.

\section{El modelo de Recuperación}

Posiblemente adoptar el modelo de recuperación es adoptar una forma de pensamiento en el que se focaliza la intención del profesional en trabajar, conjuntamente con el otro, en un camino hacia su bienestar más que centrarse meramente en el concepto de curación. Al no confrontarse un binomio enfermedad-cura, sino un binomio enfermedad-bienestar, los planes terapéuticos, se pueden centrar no tanto en una reducción de síntomas a toda costa; sino, en una escucha más profunda de los intereses, valores $\mathrm{y}$ deseos que faciliten un cambio vital. Supone de alguna forma aceptar el riesgo y los fallos. La dignidad de las decisiones que se toman y que se han tomado y el derecho a las mismas. Al adoptar esta visión se cuestiona el concepto más clásico de conciencia de enfermedad. Ello nos libera, en parte, de fútiles discusiones acerca de aspectos (que ni siquiera tenemos claros en su evidencia) sobre la etiología de la "enfermedad", para permitirnos poder hablar sobre como ha impactado en su existencia, de los aspectos traumáticos de la misma y de como hacer de la recuperación algo que merezca la pena. Esta es una reflexión importante porque las experiencias de hospitalizaciones previas y el diagnóstico de enfermedad mental suelen ser referidos una y otra vez como un elemento central en su experiencia de exclusión. Rechazan el diagnóstico, lo que a la vez les permite sobrevivir mejor (siendo el excéntrico y el raro, mejor que el loco) pero les hace rechazar posibles "ayudas" relacionadas con la enfermedad; por ejemplo, medicación o trámites burocráticos para una pensión económica por discapacidad, etc. Trabajando desde la recuperación, la enfermedad se puede convertir en un aliado del cambio.

\section{Composición y estructura del Equipo}

A fecha de hoy el equipo es multidisciplinar. Integrado por 15 profesionales y dividido en dos subequipos que trabajan como un equipo único con subdivisión de tareas y funciones. La organización de las tareas del equipo se realiza mediante reuniones de equipo de carácter semanal y en las que se comentan las incidencias de las distintas rutas y con los distintos pacientes y los objetivos que hay que priorizar. El equipo está formado por: 4 enfermeros, 2 Trabajadoras sociales y 2 psiquiatras, de los cuales uno realiza función de coordinador (Consejería de Sanidad) y 5 educadores, una trabajadora social y una psicóloga (PRISEMI). Se cuenta con recursos en los que apoyarse para su reinclusión en el sistema sociosanitario en forma de 17 plazas de 
minirresidencia, 30 de Centro de Día y 6 de pensiones.

El subequipo de enganche realiza las tareas relacionadas con el primer contacto y acercamiento en calle, enganche, mejora en el autocuidado (salud, higiene, etc), cobertura de necesidades básicas en coordinación con otros equipos y recursos que trabajan con PSH. Se desarrolla trabajo en conciencia de enfermedad y administración y supervisión de tratamiento farmacológico, intervención en crisis y mediación con el entorno, entrenamientos concretos (transporte, autocuidados, etc) y acompañamiento. Esta formado por 4 enfermeras, dos trabajadores sociales y dos educadores y cuentan con el apoyo de ambos psiquiatras tanto en trabajo de calle como en instituciones. Esta parte del equipo va a la calle casi todo el tiempo y diariamente. El avance en esta parte de la intervención suele relacionarse con estar ahí y responder a pequeñas demandas mientras se afianza la relación y a responder rápidamente a situaciones de crisis. El tiempo es rápido, las intervenciones cambiantes, exigentes. Los profesionales enfrentan decisiones ambiguas como ingresar o no; o respetar decisiones como permanecer en calle a bajo cero o en zonas peligrosas y hasta cuando hacerlo. El pacto y la negociación flexible son elementos básicos en esta etapa; así como, el acompañamiento y la mediación con otros profesionales y personas.

El subequipo de rehabilitación. El fin último de este subequipo es facilitar que la persona pueda acceder a un alojamiento y/o a una mayor autonomía cuando ya se ha conseguido una vinculación estable y unos mínimos en la intervención y la persona puede y desea seguir avanzando para conseguir una mejor calidad de vida. Se interviene principalmente en recursos de baja exigencia, albergues $u$ otro tipo de alojamientos (por ejemplo: pensiones). Habitualmente las personas reciben ya tratamiento farmacológico pero no se considera una condición indispensable, tampoco es un criterio de exclusión los consumos; si se requiere que el funcionamiento cognitivo sea suficiente para pactar la intervención y que las conductas disruptivas no supongan un riesgo o una alarma para terceros; por ejemplo, los dueños de pensiones donde puedan vivir. Está formado por una psicóloga, trabajadora social y tres educadores sociales. Trabajan de forma coordinada con los psiquiatras y los otros miembros del equipo, pudiendo mantenerse durante tiempo su función de entrenamiento y soporte aunque estén integrados en los servicios de salud mental normalizados de la red y atendidos por otro personal sanitario, hasta que se considere que se pueden desvincular de forma completa.

Los tiempos son largos y la intervención más estructurada y similar a la de un equipo de rehabilitación habitual. Sin embargo, al ser un equipo capaz de costear pensiones o de realizar búsquedas de alojamiento o recursos incluye un elemento que es la creación de un espacio. De alguna forma es más que una rehabilitación, una "habilitación" de espacios externos, un entrenamiento o recuperación de las habilidades y de rutinas para habitarlo y un trabajo interno que permita que abandone una identidad sin hogar para tener una identidad "con hogar". Es decir, que internamente pueda tolerar las dificultades de vivir fuera de la calle. Tolerar los horarios, las rutinas, la soledad, compartir el espacio, recuperar el ocio, administrar el dinero etc. Este es un trabajo costoso que obliga a afrontar miedos y renuncias. Con frecuencia, se provocan crisis o abandonos de alojamiento. La mediación y la supervisión son básicos en este subequipo.

\section{Proceso de Intervención}

La puerta de entrada al programa, excepto situaciones atípicas como autoderivaciones o peticiones desde el sistema judicial, por parte de los servicios sociales específicos es una reunión mensual (mesa de derivación). En esta reunión se comentan los casos, se prioriza su atención y se define la forma, lugar y el momento más adecuado para el primer contacto. La derivación de los casos que son detectados por el sistema sanitario (unidades de agudos de los hospitales generales) se realiza por contacto telefónico con el coordinador del programa y tras acudir al hospital a conocerlo/a.

Contacto inicial. En la calle. Este contacto se realiza en compañía del trabajador social de referencia y/ o cualquier persona con la que tengan un vínculo seguro si es que este existe. Esta primera entrevista es llevada a cabo por las psiquiatras. $\mathrm{Su}$ objetivo es conseguir una primera aproximación diagnóstica y, ante todo, crear un clima que favorezca nuevas visitas. También es su objetivo descartar que exista una patología física que suponga un riesgo vital inminente o que exista un riesgo para sí mismo o para terceros. Ello podría suponer una indicación de ingreso urgente y rediseñar el abordaje incluyendo al hospital.

Cuando el primer contacto se efectúa en el hospital se intenta favorecer el enganche y recabar información sobre que recursos o zonas utilizan habitualmente por si resultan difíciles de ubicar o si se "pierden" tras el alta. Se intenta pactar el siguiente contacto y plan terapéutico al alta que acepta el paciente (si es que acepta alguno) o simplemente se les facilita la manera de contactar en caso de no aceptar otra alternativa. Se pretende así que exista una continuidad de cuidados sea en la calle o en el albergue al que sean derivados si carecen de contacto previo con los servicios de salud mental. Es muy habitual tener que lidiar con diferencias de opinión con el personal sanitario del hospital sobre cuando y como se debe de realizar el alta. Se procura que el alta se coordine para que la persona; al menos, pueda tener plaza en un albergue o centro de baja exigencia y que pueda ser acompañado, en el momento del alta, a la salida de su hospital o acogerles en su llegada al alojamiento al que vayan o acompañarles su lugar en la calle.

Trabajo de calle. Tras la entrevista inicial se mantienen varias entrevistas diagnósticas y de presentación del resto del equipo. La decisión de que profesionales se harán cargo de la intervención se realiza en las reuniones de equipo; en función a la carga de trabajo, afinidades personales o los conocimientos (muchas veces extracurriculares, como el interés por la música o el deporte) de los miembros del equipo. Se realiza una valoración de enfermería y social. Se intenta promover un cambio en los aspectos de salud y de autocuidados que puedan suponer una mejora en su calidad de vida. El seguimiento se efectúa dónde se encuentren los pacientes: en la vía pública, en 


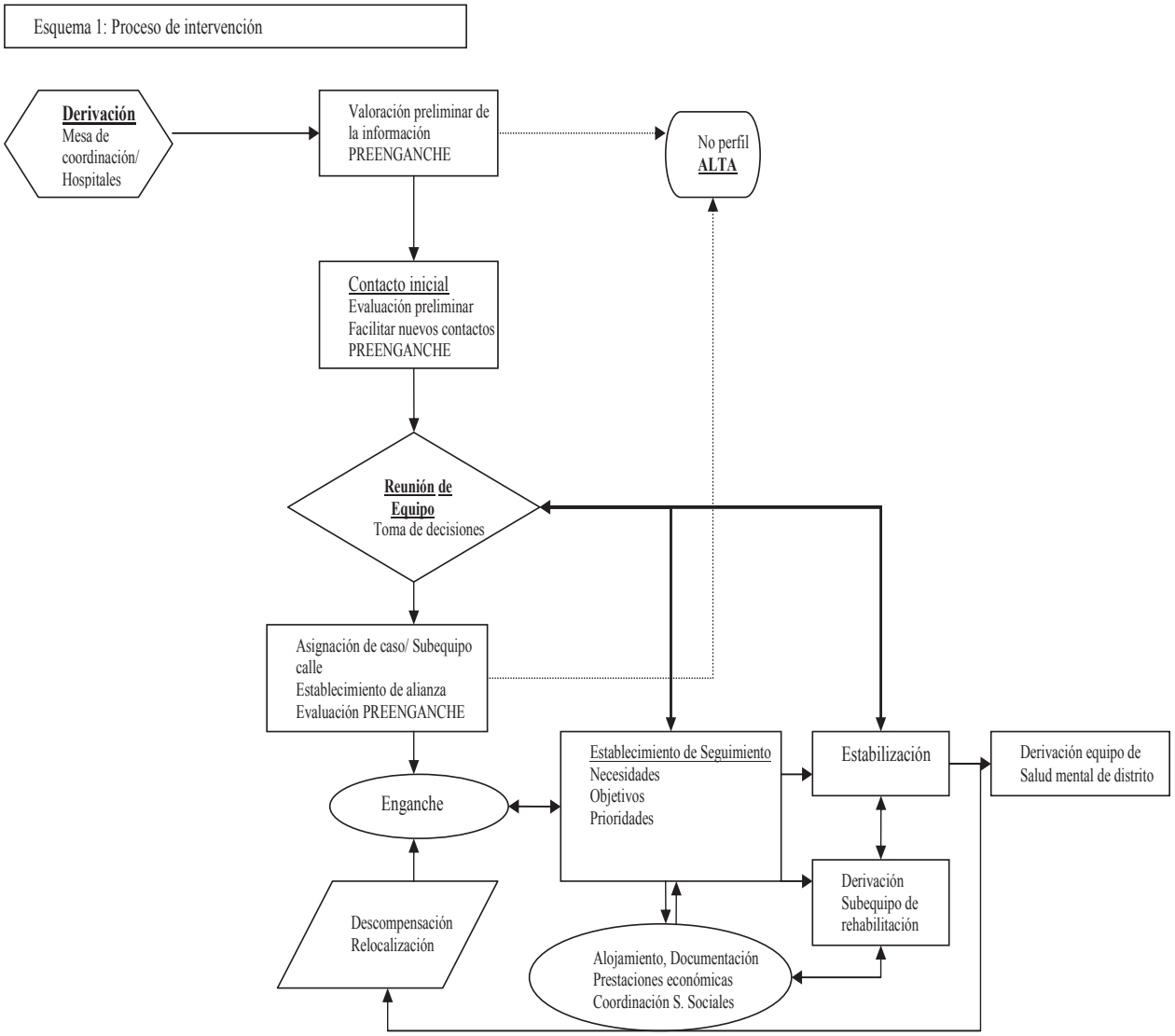

Figura 1. El proceso de intervención

los recursos sociales y en consulta, en aquellos que aceptan y pueden acudir a la misma. En general, se intenta mantener un contacto mínimo semanal, por parte de un miembro del equipo. El horario y la periodicidad se modifica para adaptarse a las costumbres (horarios de comedor, lugares donde piden, horas de mayor o menor intoxicación etílica) respetando e intentando no interferir en rutinas importantes y dependiendo del momento de la intervención (por ejemplo, supervisión diaria en pacientes que inician tratamiento farmacológico) o sus necesidades (por ejemplo, gravedad del estado físico) o incluso las inclemencias climáticas (por ejemplo, riesgo de congelación/deshidratación. En general, las visitas las realizan al menos dos miembros del equipo para poder dar respuesta a situaciones de urgencia o, en ocasiones, al entorno (por ejemplo, viandantes, vecinos). Se respetan los límites de acercamiento del paciente y ante riesgo de violencia o incomodidad del paciente se limita el tiempo de la intervención. Es frecuente que durante los momentos iniciales de enganche las entrevistas sean cortas e informales, con temas más o menos circunstanciales. Lo principal es conseguir una comunicación que vaya facilitando el vínculo y permita ir avanzando por diferentes "estadíos de cambio" (Prochaska \& Prochaska, 2001); conocer su lenguaje verbal y gestual y poder ir pactando objetivos y su mantenimiento. Simultáneamente se suelen ir trabajando, en coordinación con otros recursos, la obtención de documentación, tarjeta sanitaria, pensiones no contributivas, rentas mínimas o alojamiento si así lo demandan.

En la calle se podemos realizar cualquier intervención que se pueda prestar en un Centro de salud mental con enfermos graves, excepto realizar analíticas. La administración de tratamiento farmacológico se realiza con cuidado. Se usan fármacos en monoterapia, titulando despacio las dosis desde dosis muy bajas, con supervisión diaria en calle y en algunos recursos o asumiendo administrar tratamientos solo de manera puntual para reducir el malestar. Se intenta evitar que la toma de medicación suponga un riesgo mayor (sedación) o que interfiera en su estilo de vida. Los profesionales que trabajan en calle no van uniformados, ni se les puede identificar como médico, psiquiatra o enfermera. Esto no es azaroso, sino un posicionamiento para evitar una señalización mayor en el entorno y los problemas que puede ocasionar a la PSH; aunque, a veces, es un problema de cara a policía o incluso vecinos.

Trabajo en instituciones y SSM. Se realiza enganche y seguimiento en albergues, centros de acogida $u$ otros recursos sociales. Se mantiene el seguimiento de pacientes previamente contactados con en equipo cuando son alojados o cambian de alojamiento y durante la transición hasta un recurso normalizado por parte de los mismos profesionales que le acompañaron en calle. Si se mantiene estable en el recurso se valora si la intervención debe de ser asumida por el equipo de rehabilitación, 


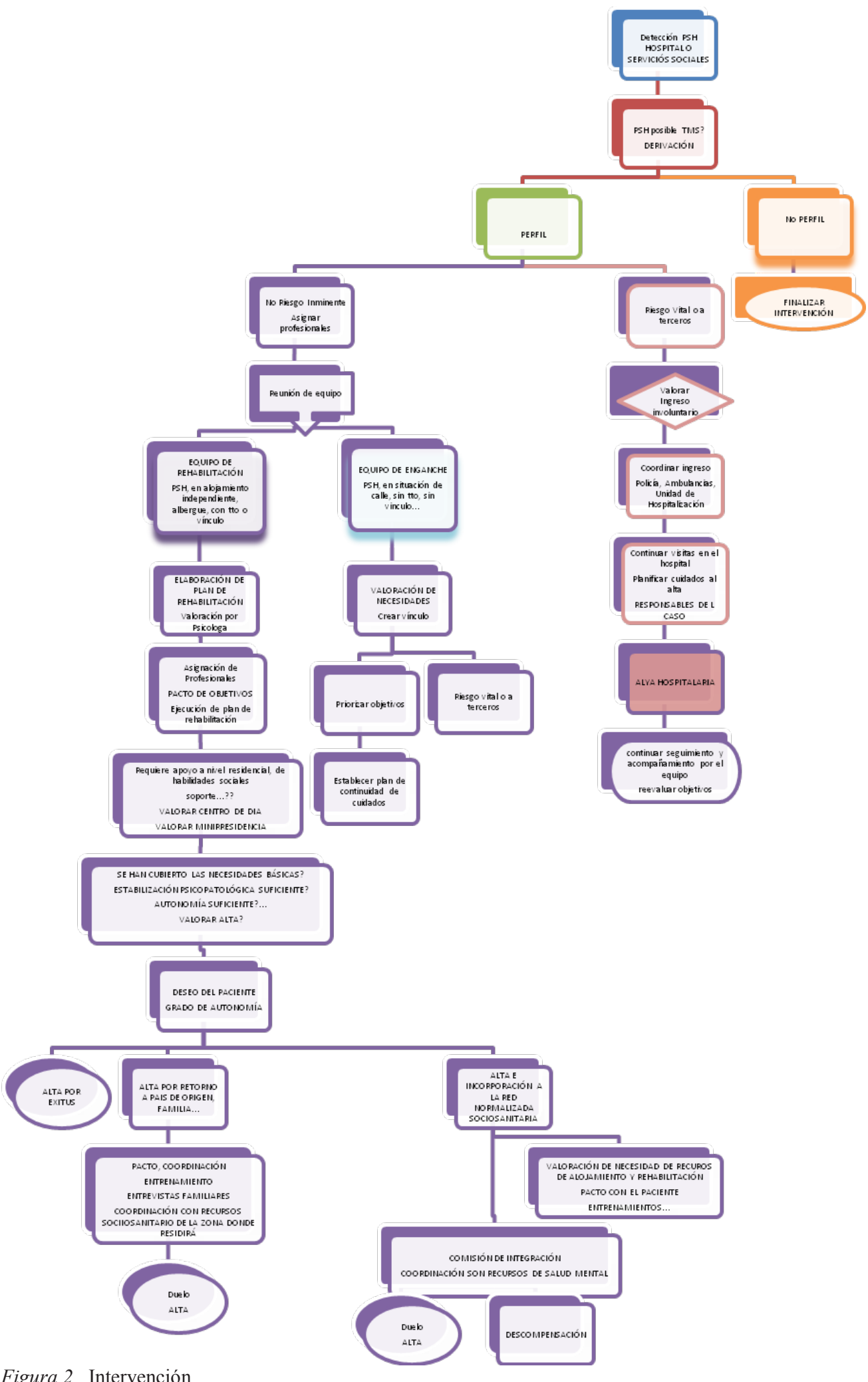

desde el centro de día o vinculándolo a recursos normalizados. Se efectúan intervenciones en situaciones de crisis "in situ" si se precisa o entrenamientos concretos (transporte, conocimiento del entorno, acompañamientos) para que puedan transitar a otro recurso o administración de medicación. Se cumple una función de asesoramiento o apoyo y mediación con personal de dichos centros con una periodicidad variable (semanal o quincenal). Esta última actividad suele ser más una interconsulta con el psiquiatra o el coordinador del equipo. La función del seguimiento es establecer y reafirmar el vínculo con el paciente, hacer valoraciones diagnósticas, conseguir que acepten tratamiento y alojamiento, favorecer el autocuidado, la transición a otros 
alojamientos o que acudan a la consulta.

Atención ambulatoria en el servicio de salud mental de centro. Semanalmente hay un día de consulta (medico y de enfermería) abierto, sin agenda en el que cualquier paciente puede acceder con o sin cita previa y en el que se garantiza que se les atenderá. No se les demanda ninguna documentación, ni abstinencia de tóxicos y se les invita acudir no sólo en función del seguimiento sino también como una referencia a donde ir si tienen alguna emergencia o problema psíquico, físico o social. En caso de pacientes intoxicados se modifica la entrevista haciéndola más breve e informal y facilitando otra cita para poder hablar de lo que les preocupa.

Acceso a recursos y a la atención normalizada. Una vez que se han conseguido unos objetivos mínimos o a petición directa de los usuarios se planifica su entrada a los servicios de salud mental y a los recursos (minirresidencias, piso supervisados) de la red sociosanitaria integrada. No hay un tiempo prefijado de duración de la intervención. El carácter de transición o puente del programa asienta en razones prácticas e ideológicas. La primera es que el programa se saturaría y dejaría de cumplir su función de trabajo asertivo e intensivo. La segunda es defender que las PSH tienen derecho a acceder a las mismas prestaciones que el resto de la población y que es el sistema el que tiene que flexibilizar sus barreras y objetivos para incluirlos. De manera formal, la entrada al sistema sanitario se hace a través de una comisión de integración, bimensual, coordinada desde la oficina de coordinación regional de salud mental. A esta comisión se citan a los diversos servicios de salud mental y se les comentan los posibles recursos e intervenciones a emplear para que las personas puedan incluirse con garantías en la red. Estos planes son pactados con los pacientes antes de iniciar un desenganche. Sería ideal decir que se respeta ante todo la opinión y deseos de los pacientes. En realidad, la decisión de a donde derivarles es compleja y aunque depende de que ellos lo acepten o no, está limitada (en gran medida) por los recursos que están disponibles en la red normalizada y de la saturación de los mismos. Se negocia el paso a la red de salud mental y; con frecuencia, las reticencias de los profesionales a admitir a estas personas. Estas reticencias suelen relacionarse con el escepticismo ante la actuación de un equipo de salud desconocido y un poco "atípico". Por el consumo de recursos, habitualmente escasos para la población que conocen y sienten como suya; o simplemente, por el reconocimiento de que los recursos a emplear no son completamente adecuados para estas personas.

Por parte de nuestro equipo el desenganche es posiblemente la parte peor resuelta de la intervención. Se suelen activar toda suerte de duelos y temores. El más frecuente, es si los otros equipos van a atender "bien" a "nuestro" paciente, si volverán a la calle, si les dejamos solos. Se mezcla el temor a la independencia del otro, de decisiones sobre las que no tenemos control y sobretodo, una crítica más o menos solapada hacia los recursos normalizados. Trabajar en un equipo como este tiene un alto gasto emocional, se abordan de forma continuada problemas y situaciones límite (violencia, abandono, agresiones, muerte). Para tolerarlas, el apoyo de otros miembros y la cohesión del equipo se vuelve indispensable. En momentos de gran tensión con el sistema esto se convierte en una identidad de equipo construida desde la diferencia o desde el enfrentamiento. De alguna forma proyectamos lo que no podemos tolerar, como sentimientos de culpa, inutilidad, vulnerabilidad o rabia. La ausencia de una supervisión institucional se hace muy patente en estos momentos. Técnicamente es trabajar un duelo y establecer una retirada progresiva de los profesionales y sus apoyos. Igual que su incorporación fue progresiva, se retiran antes los profesionales cuya función pueda ser reemplazada con más rapidez por otros profesionales de la red normalizada y más lentamente, los que pueden realizar un apoyo más efectivo. Esto se puede mantener de forma intermitente, si hace falta, y de manera informal durante años, aún estando incluidos en un servicio de salud mental, sin otra función que el apoyo.

En esta etapa final, cada vez es más frecuente nos encontrar que los problemas judiciales y los problemas relacionados con la situación irregular de muchos de nuestros pacientes son el factor más limitante de la rehabilitación. En un número no desdeñable de casos surge, de repente, una condena o un problema legal desconocido o una orden de expulsión que paraliza toda posible reinclusión. En muchos casos, la defensa legal no fue eficaz, o la enfermedad mental provocó la condena o la falta de una apelación A fecha de hoy no podemos ofertarles un apoyo legal suficiente.

\section{Resultados}

En la última revisión sistemática de datos realizada en el 2009, incluyendo los datos desde el inicio del programa hasta finalizar ese año, se habían atendido a 393 personas. Entre ellas existía un predominio de varones, de edades medias de la vida. La gran mayoría no tenían pareja o relaciones afectivas relevantes o no se conocía ese dato. Las fuentes de ingresos más frecuentes eran o carecer de ellas, ayudas estatales que les habíamos tramitado o la mendicidad. Entre las mujeres había un número mayor de personas que ejercían la prostitución como fuente económica. El diagnóstico más frecuente era de Esquizofrenia (57\%) muchos de ellos duales (alcohol o alcohol- cannabis). Durante el tiempo de intervención de nuestro equipo un $68 \%$ realizaban tratamiento farmacológico. Al hablar de alojamientos las cifras se acercaban a un $80 \%$ de personas fuera de la calle. Tras la intervención, con cifras de 200 pacientes dados de alta, un $31 \%$ estaban vinculados a SSM, un 25\% habían retornado a su país o ciudad de origen, y un $4 \%$ con sus familias. Un $2 \%$ estaban en residencias, no dependientes de la red de salud mental. Un $15 \%$ de las personas se les dio de alta por no tener perfil para ser atendidos en el y un 3\% se revincularon con MAP y no con servicios de salud mental. Tristemente un $1 \%$ estaban en la cárcel (cifra que está aumentando) y 23 personas habían fallecido durante la intervención o tras el alta. Esto destaca que es un trabajo útil pero con muchas cosas a mejorar. Destacar por su importancia, la salud física, lo legal y la atención a personas inmigrantes en situación irregular. 


\section{Referencias}

Brandt, P. (2003). Encuentro con los enfermos mentales que viven en la calle. Cuadernos de Psiquiatría Comunitaria, 3(2), 148-157.

Bravo Ortiz, M. F. (2003). Intervenciones en personas sin hogar con trastornos mentales: Situación en Europa y en España. Cuadernos de Psiquiatría Comunitaria, 3(2),117-128.

Chinman, M. J., Rosenheck, R., \& Lam, J. A. (1999). The Developing of Relationships between People who are Homeless and have Mental Disability and their Case Manangers. Psychiatric Rehabilitation Journal, 23(1), 47-56.

Dixon, L. (2000). Assertive Community Treatment: twenty- five years of gold. Psychiatric Services, 5(6), 759-765.

Faris, R. E. L., \& Dunham, H. L. (1939). Mental disorder in urban areas. Chicago: Chicago University Press.

Levy, J. (1998). Homeless outreach: A developmental model. Psychiatric Rehabilitation Journal, 22(2),123-132.

Martínez Hernandez, A. (1998). ¿Has visto como llora un cerezo? Pasos hacia una antropologia de la Esquizofrenia. Barcelona: Universitat de Barcelona.

Prochaska J. M., \&Prochaska, J. O. (2001). ¿Porqué no se mueven los continentes? ¿Porqué no cambian las personas? Revista de psicoterapia, 12(46/47), 17-36.
Rodríguez, A., \& González Cases, J. C. (2002). La rehabilitación psicosocial en el marco de la atención comunitaria integral a la población enferma mental crónica. In Consejería de Servicios Sociales de la Comunidad de Madrid (Org.), Rehabilitación psicosocial y apoyo comunitario de personas con enfermedad mental crónica: programas básicos de intervención (pp. 1742). Madrid: Autor.

Salud Mental Exclusión Social [SMES] (2001). Propuesta de Actuación Coordinada en la Atención a Personas Sin Hogar con Trastornos Mentales. Boletin de la Asociación Madrileña de Salud Mental, primavera, 5-15.

Teicher, M. H., Andersen, S. L., \& Polcari, A. (2003). The neurobiological consequences of early stress and childhood maltreatment. Neuroscience and Behavioural Rewievs, 27, 33-44.

Test, M. A., \& Stein, L. I. (1976). Practical guidelines for the community treatment of markedly impaired patients. Community Mental Health Journal,12(1), $72-82$.

Vega González, L. S. (1996). Salud Mental en Población sin Hogar. Cuadernos Asturianos de Salud. Oviedo: Servicio publicaciones.

Wright, C., Burns, T., James, P., \& Billings, J.(2003). Assertive outreach teams in London: models of operation. Pan-London Assertive Outreach Study, part 1. British Journal of Psychiatry, 183, 132-138.

1. Ambos aspectos en mayor o menor grado existen a lo largo de la existencia de muchas personas que padecen enfermedades mentales graves o en sus familias. De hecho, son un colectivo en frecuente riesgo de exclusión debido al aislamiento, a la marginación o a las dificultades para conseguir una participación social plena. Pocos acceden al mercado laboral, quedan aislados o a cargo de sus familias y, con frecuencia, sus decisiones son cuestionadas o simplemente ignoradas en base a su enfermedad aún cuando no estén relacionadas con ellas. Recientemente a una de las personas que atiendo su hematólogo le remitió a mí para que le explicara que tenía un cáncer hematológico porque "no lo iba entender"; como le explicó "amablemente" a la educadora que le acompañaba y tras explicarle a ella (delante de él) su enfermedad. La preocupación de mi paciente y por la que no había firmado en inicio el consentimiento informado para sus pruebas era el dolor y su temor a padecerlo. Tras asegurarle que le pautaría toda la analgesia que precisara y que le acompañaría a las pruebas lo firmó sin problema. No había ningún elemento paranoide en todo su discurso, ni ninguna merma en su capacidad de comprender.

2. Sin techo suele hacer referencia a aquellas personas que ni siquiera utilizan recursos ni para dormir o comer. Toda su actividad vital incluido dormir, lavarse... lo hacen en la calle.

3. En el caso de la población sin hogar, a veces, la carencia de otros informadores y el desconocimiento del curso de la enfermedad y la trayectoria vital hacen que los criterios de discapacidad y el no acceso a la red normalizada (o su negativa a acudir a ella) sean con frecuencia más importantes que el propio diagnóstico o la duración del trastorno.

4. Lo que perciben más inicialmente en los albergues y centros residenciales que en la propia calle.

5. El Samur Social se ocupa de las emergencias sociales y personas sin hogar. Está constituido por una central y unidades móviles que coordina las emergencias y centraliza las demandas e informaciones de la población; y los equipos de calle. Los equipos de calle (constituidos por auxiliar y trabajador social) son los encargados de la prospección y detección de las personas sin hogar. Realizan el primer acercamiento y cubren todas las actividades para acompañar o facilitar la salida de estas personas de la calle.

6. Es frecuente que lleguen a urgencias o a una consulta y se le pueda llegar a atender "bien" la enfermedad e indicar un fármaco antipsicótico. "3 veces al día, con las comidas y este cuando se vaya a dormir". Buena práctica, sin duda cuando se come 3 veces al día cada 6 u 8 horas, respetando la vida media del fármaco y se indica el más sedativo para facilitar el descanso. Pero ¿y si comes al ritmo de un comedor, con cena a las 7 y comida variable?, ¿la tomas o no? Y sedarse, a bajo cero y en una acera posiblemente suponga un riesgo para tu vida (congelación...) o al menos que te puedan robar; y sin documentación ¿cómo se pide la siguiente cita? Sin dinero ¿cómo pagas la medicación?

Maria Isabel Vázquez Souza é psiquiatra do Servicio Madrileño de Salud/Hospital Clínico Universitario San Carlos e coordenadora do Programa de Atención Psiquiátrica dirigido a Enfermos Mentales sin Hogar. Endereço para correspondencia: Centro de Salud Mental. Distrito Centro. C/Cabezas 4. Madrid. 28012. Telefone: ++34 699945251. E-mail: mvazquez.scsm@salud.madrid.org 